\title{
THE EFFECTS OF PROSTHETIC CARDIAC BINDING AND ADYNAMIC CARDIOMYOPLASTY IN A MODEL OF DILATED CARDIOMYOPATHY
}

Joong Hwan Oh, MD

Vinay Badhwar, $\mathrm{MD}^{\mathrm{b}}$

Brian D. Mott, MD ${ }^{\mathrm{b}}$

Carlos M. Li, MD ${ }^{\mathrm{b} *}$

Ray C.-J. Chiu, MD, $\mathrm{PhD}^{\mathrm{b}}$
Objective: Because adynamic cardiomyoplasty, or wrapping skeletal muscle around the heart, had been shown to provide a girdling effect and delay progressive ventricular dilatation in heart failure, a similar girdling effect by the much simpler procedure of cardiac binding, using a prosthetic membrane to wrap the heart, was studied and compared with that of adynamic cardiomyoplasty. Methods: Twenty-one dogs were divided into control, adynamic cardiomyoplasty, and cardiac binding groups. Cardiac dimension and hemodynamic studies were carried out before and 4 weeks after rapid pacing at $\mathbf{2 5 0}$ beats/min. For adynamic cardiomyoplasty, the left latissimus dorsi muscle was used for the cardiac wrap; for cardiac binding, a Marlex sheet (C. R. Bard, Inc., Murray Hill, N.J.) was used. Serial two-dimensional echocardiography, right heart catheterization, and in the cardiac binding group, left heart catheterization were performed. Results: Four weeks of rapid pacing induced severe heart failure and cardiac dilatation. The magnitude of ventricular dilatation at the end of rapid pacing was less in the cardiac binding group than in the control group and least in the adynamic cardiomyoplasty group. Left ventricular end-diastolic volume, end-systolic volume, and ejection fraction were $82.1 \pm 21.1 \mathrm{ml}$, $67.1 \pm 16.0 \mathrm{ml}$, and $\mathbf{1 7 . 5 \%} \pm \mathbf{5 . 8 \%}$, respectively, in the control group; 61.9 . $\pm 8.1 \mathrm{ml}, 44.1 \pm 7.8 \mathrm{ml}$, and $30.1 \% \pm 3.6 \%$, respectively, in the cardiac binding group; and $51.8 \pm 8.7 \mathrm{ml}, 30.3 \pm 10.4 \mathrm{ml}$, and $27.0 \% \pm 4.0 \%$, respectively, in the adynamic cardiomyoplasty group. Conclusions: Both adynamic cardiomyoplasty and cardiac binding reduced cardiac enlargement and functional deterioration after rapid pacing, with adynamic cardiomyoplasty appearing to be more effective, perhaps because of the adaptive capabilities of the skeletal muscle wrap. However, cardiac binding is a simpler and less invasive procedure, which may be useful as an adjunct to prevent or delay progressive ventricular dilatation in heart failure. (J Thorac Cardiovasc Surg 1998;116:148-53)
From the Division of Cardiothoracic Surgery, McGill University, Montreal, Quebec, Canada, ${ }^{\mathrm{b}}$ and Department of Thoracic and Cardiovascular Surgery, Yonsei University, Wonju College of Medicine, Wonju City, Republic of Korea. ${ }^{a}$

This work was supported by an operating grant from the Medical Research Council of Canada.

Received for publication June 27, 1997; revisions requested Sept. 15, 1997; revisions received Feb. 4, 1998; accepted for publication Feb. 4, 1998.

Address for reprints: Ray C.-J. Chiu, MD, The Montreal General Hospital, 1650 Cedar Ave., Room C9.169, Montreal, Quebec, Canada H3G 1A4.

*Research Fellow of the Canadian Heart and Stroke Foundation.

Copyright (C) 1998 by Mosby, Inc.

$0022-5223 / 98 \$ 5.00+0 \quad \mathbf{1 2} / \mathbf{1} / \mathbf{8 9 4 5 4}$
Uurgical reduction of cardiac size to improve heart failure in patients with dilated cardiomyopathy is being popularized by Batista and associates ${ }^{1}$ with the partial ventriculectomy operation. Although early increase in ejection fraction has been noted in many surviving patients, ${ }^{2,3}$ the long-term outcome after this operation is still unknown. One of the concerns is the progressive redilatation of the ventricle. It is reasonable to speculate that measures to prevent or delay such progression of ventricular dilatation could be helpful. We suggest that a "cardiac binding procedure" may be useful for this purpose.

Our concept of using prosthetic material to wrap around the ventricles to reduce ventricular dilata- 


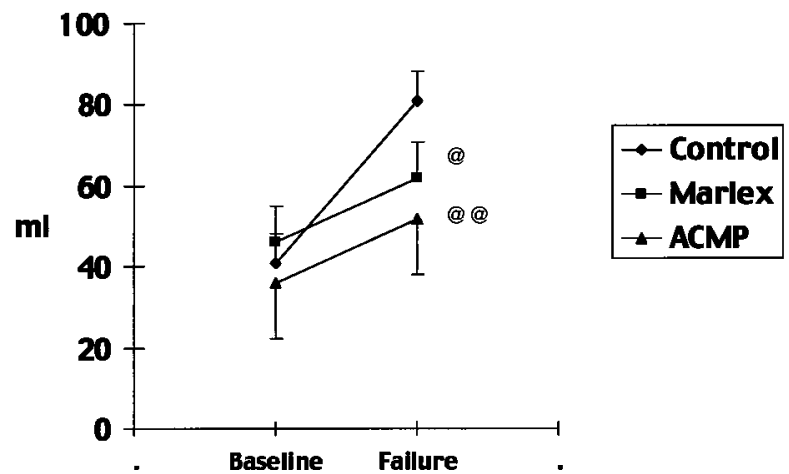

Fig. 1. The cardiac binding (Marlex) and adynamic cardiomyoplasty $(A C M P)$ groups showed less end-diastolic ventricular dilatations at the end of 4 weeks of rapid pacing than the control group $(p=0.041 ; p=0.017)$.

tion in a failing heart is an offshoot from our study on the mechanisms of dynamic cardiomyoplasty, a procedure that is currently undergoing a phase III prospective randomized clinical trial..$^{5}$ These studies have shown that although dynamic contractions of skeletal muscle wrapped around the ventricles in synchrony with cardiac systole can reduce myocardial stress and may improve systolic function, the passive, that is, adynamic, wrapping of the failing ventricles with skeletal muscle may by itself reduce the progression of ventricular dilatation. ${ }^{6}$ Such observation in adynamic cardiomyoplasty has been called "the girdling effect."7 To further elucidate the mechanisms of the girdling effect, we compared adynamic cardiomyoplasty with a cardiac binding procedure. In the latter, a prosthetic membrane such as Marlex mesh (C. R. Bard, Inc., Murray Hill, N.J.) is used to wrap the ventricles. The effects of these procedures in a canine model of rapid-pacing heart failure were compared. The results indicate that cardiac binding may have a role in the management of selected patients with heart failure.

\section{Materials and methods}

Twenty-six mongrel dogs weighing 23 to $33 \mathrm{~kg}$ (mean $27.9 \pm 3.2 \mathrm{~kg}$ ) were studied in accordance with the Guidelines of the Canadian Council for Animal Care. The animals were randomly allocated into three groups. Five dogs were eliminated from final data analyses for the following reasons: Two (one each in the adynamic cardiomyoplasty and cardiac binding groups) had faulty, improperly pacing pacemakers; two (one each in the adynamic and binding groups) died suddenly of presumed arrhythmias; and another in the binding group died of thromboembolism after cardiac catheterization. In the control group $(n=7)$ a pacemaker was inserted 10 days before the onset of rapid pacing. The rapid pacing was continued

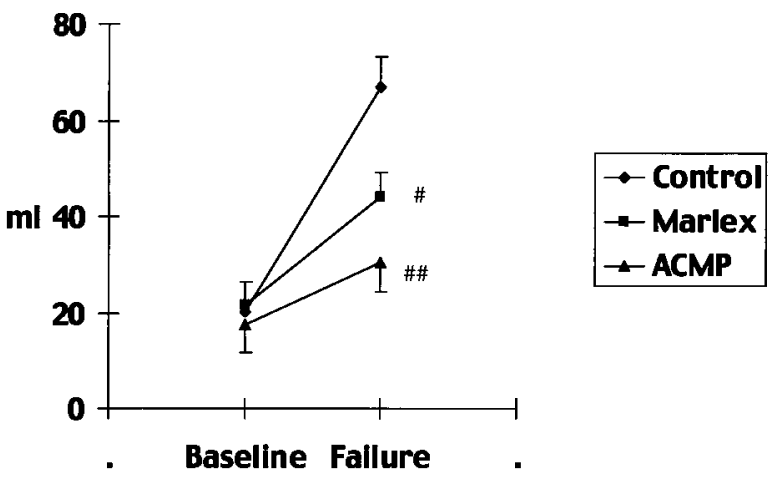

Fig. 2. The cardiac binding (Marlex) and adynamic cardiomyoplasty $(A C M P)$ groups showed less end-systolic ventricular dilatation at the end of 4 weeks of rapid pacing than the control group $(p=0.007 ; p=0.001)$.

for 4 weeks to induce severe heart failure. In the cardiac binding group $(n=7)$, the same protocol as that for the control group was followed, except that both ventricles were wrapped with Marlex mesh at the time of the pacemaker implantation before rapid pacing. In the third group, adynamic cardiomyoplasty $(n=4)$ was carried out by using the left latissimus dorsi muscle to wrap the heart. In this group, the adynamic cardiomyoplasty procedure was done 4 weeks before the onset of rapid pacing so that the animals could recover from the more invasive procedure and to allow the wrapped skeletal muscle to undergo "conformational change," as will be discussed later. Then these animals also underwent 4 weeks of rapid pacing.

Cardiac binding. Anesthesia was induced with sodium pentobarbital $(30 \mathrm{mg} / \mathrm{kg})$ and maintained with $1 \%$ isoflurane and oxygen delivered by a mechanical ventilator (Muffled Anesthesia Ventilator series 200, Intermed, Penlon, United Kingdom). A median sternotomy was performed for pacemaker insertion and for cardiac binding with Bard Marlex mesh. A wide pericardiotomy was carried out and the Marlex mesh was wrapped around both ventricles below the atrioventricular groove, snugly against the epicardium but without tension, and sewn to itself anteriorly to complete the wrap.

Adynamic cardiomyoplasty. Under general anesthesia, the left latissimus dorsi muscle was harvested with the animal in the right lateral decubitus position. The distal and proximal muscular tendinous attachments were divided and the thoracodorsal neurovascular pedicle was preserved. No cardiomyostimulator was implanted. The muscle was then transposed into the left pleural cavity through a minithoracotomy created by resection of the second rib anteriorly. The proximal tendon was reattached to the periosteum of the resected rib to anchor it in place, taking care not to compromise the thoracodorsal nerve and vessels. The incision was closed in layers. The animal was then repositioned in the supine position and a median sternotomy performed. A wide pericardiotomy was carried out and a pacemaker for rapid pacing was implanted as described earlier. After this, the left latissimus dorsi muscle was retrieved from the pleural space and wrapped 


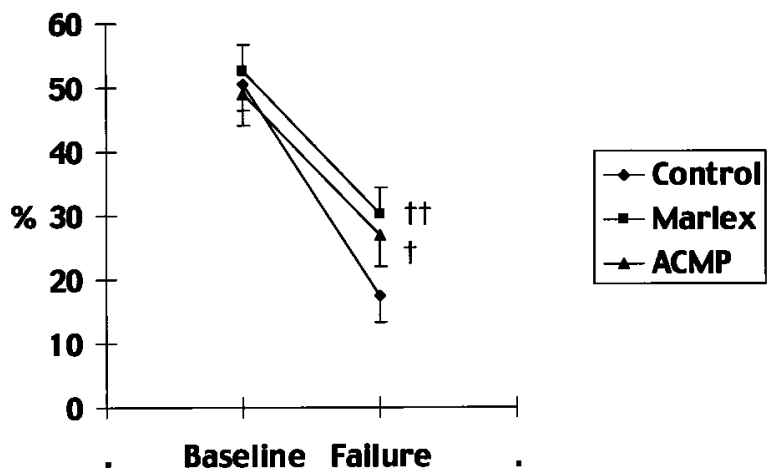

Fig. 3. Ejection fractions decreased at the end of rapid pacing in all groups. The cardiac binding (Marlex) and adynamic cardiomyoplasty $(A C M P)$ groups have higher values than the control group $(p=0.015 ; p=0.001)$.

snugly in a posterior clockwise direction around both ventricular surfaces, but without excessive tension. The muscle was sewn to the pericardium along the posterior atrioventricular groove. ${ }^{8}$ The epicardial pacing lead would be used for rapid pacing. The mediastinum was drained and the sternum closed with wires. These animals were allowed to recover for 4 weeks before rapid pacing was initiated.

Induction of heart failure. Heart failure was induced during the 4-week period of continuous rapid pacing with a modified Medtronic 8329 pacemaker (Medtronic, Inc., Minneapolis, Minn.). The pacemaker was placed in the subcutaneous pocket in the anterior abdominal wall, and a Medtronic 5071 myocardial lead was placed near the apex of the left ventricle. These pacemakers were programmed transcutaneously by telemetry to a rate of 250 beats/min with a Medtronic model 9710 programmer.

Hemodynamic studies. Cardiac dimensions were measured before and 4 weeks after the rapid pacing with a two-dimensional transthoracic echocardiogram (model 77020 AC, Hewlett-Packard Company, Andover, Mass.) with the animal under general anesthesia. Both enddiastolic volume and end-systolic volume were measured and ejection fraction was calculated. Other hemodynamic data were obtained with Swan-Ganz pulmonary catheters (Baxter Healthcare Corporation, Edwards Division, Santa Ana, Calif.). The stroke volume was measured by the thermodilution method (model 90303A, Space Labs Inc., Redmond, Wash.). Hemodynamic parameters measured included central venous pressure, right ventricular pressure, main pulmonary artery pressure, and pulmonary capillary wedge pressure. Systemic blood pressure was monitored with a femoral artery catheter. The left side of the heart was catheterized with a $5 \mathrm{~F}$ pigtail catheter (Selector, Namic Corp., Glens Falls, N.Y.) for the cardiac binding group, specifically to rule out the possibility of constrictions around the heart. During the catheterization, the left ventricular end-diastolic pressure and rate of left ventricular pressure rise were measured.

Each measurement was repeated three times. Statistical analysis was carried out by means of SPSS version 7.5

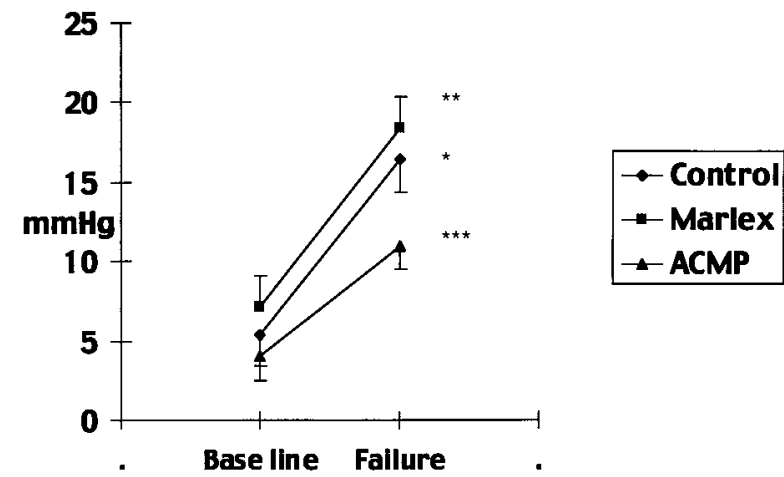

Fig. 4. Pulmonary capillary wedge pressures in all three groups during heart failure were higher than baseline ( $p<$ $0.001 ; p=0.014 ; p=0.012$.) The differences among the groups did not reach statistical significance.

(SPSS, Inc., Chicago, Ill.) with multivariate analysis of variance, treating two time points (baseline and failure) as repeated measures, in conjunction with the Scheffe test to compare the differences between the cardiac binding and adynamic cardiomyoplasty groups. The data were expressed as mean \pm standard deviation.

\section{Results}

Changes in cardiac dimensions measured by echocardiography are shown in Figs. 1 to 3. All three groups of animals had similar baseline values just before the start of rapid pacing. Four weeks of such rapid pacing resulted in severe heart failure in all three groups as reflected in increases in pulmonary capillary wedge pressure when compared with the baseline (control, $p<0.001$; adynamic cardiomyoplasty, $p=0.012$; binding, $p=0.014$ ) (Fig. 4), decreases in ejection fraction and stroke volume, and enlargement of ventricular volumes in both systole and diastole. The magnitude of ventricular dilatation at the end of the rapid pacing phase was less in the cardiac binding (Marlex mesh) and adynamic cardiomyoplasty groups than in the control group. Left ventricular end-diastolic and endsystolic volumes in the cardiac binding group were $61.9 \pm 8.1 \mathrm{ml}(p=0.041)$ and $44.1 \pm 7.8 \mathrm{ml}(p=$ $0.007)$, respectively, compared with $82.1 \pm 21.1 \mathrm{ml}$ and $67.1 \pm 16.0 \mathrm{ml}$, respectively, in the control group. Similarly, in the adynamic cardiomyoplasty group, left ventricular end-diastolic and end-systolic volumes were $51.8 \pm 8.7 \mathrm{ml}(p=0.017)$ and $30.3 \pm$ $10.4 \mathrm{ml}(p=0.001)$, respectively. Left ventricular ejection fractions were higher in the cardiac binding and adynamic cardiomyoplasty groups than in the control group. Left ventricular ejection fractions in the cardiac binding and adynamic cardiomyoplasty 
groups were $30.1 \% \pm 3.6 \%(p=0.001)$ and $27.0 \%$ $\pm 4.0 \%(p=0.015)$, respectively, compared with $17.5 \% \pm 5.8 \%$ in the control group at the end of the rapid pacing phase.

Lack of equalization of mean left ventricular end-diastolic pressure $(21 \pm 4.6 \mathrm{~mm} \mathrm{Hg})$ with right ventricular end-diastolic pressure $(15.3 \pm 1.3 \mathrm{~mm}$ $\mathrm{Hg}$ ) in four selected animals subjected to cardiac binding during the heart failure period suggested no evidence of constrictive restrictions by the Marlex mesh around the heart. However, the statistical power for this observation is limited by the small sample size.

\section{Discussion}

As stated earlier, this study was originally initiated to further elucidate the mechanisms of the dynamic cardiomyoplasty procedure. Use of dynamic cardiomyoplasty to treat heart failure has undergone phase I and phase II clinical trials, and a phase III prospective randomized study is in progress in North America. Dynamic cardiomyoplasty alleviates the symptoms of moderate to severe heart failure and improves the functional capacity and the quality of life in most treated patients, but hemodynamic changes are modest. ${ }^{5}$ These findings have led to renewed investigation on the possible underlying mechanisms of dynamic cardiomyoplasty. ${ }^{6}$ Systolic squeeze is the originally proposed, and still important, mechanism of dynamic cardiomyoplasty. For example, recently a new technique in which a balloon was placed in the interface between the skeletal muscle wrap and the epicardium demonstrated that the transmyocardial pressure gradient decreased in dynamic cardiomyoplasty when the wrapped muscle was stimulated to contract in synchrony with cardiac systole. ${ }^{10}$ This leads to reduced wall stress during systole, known as the "myocardial sparing effect." However, long-term follow-ups of these patients indicate that there may be other additional effects of cardiomyoplasty. Serial radiographic studies show that in many patients the cardiothoracic ratio stabilized, ${ }^{11}$ which is confirmed by echocardiography and by ventricular pressure-volume loops obtained with conductance catheters. In some patients, the cardiac size appeared to decrease over time, which was described as "reverse remodeling" by Kass and colleagues. ${ }^{12}$ Of particular interest was the observation that in one of their patients, despite the lack of demonstrable contraction of the wrapped muscle, perhaps owing to technical problems, the modulation of the myocardial remodeling process seen in heart failure appeared to take place in the presence of the passive muscle wrap, that is, "adynamic cardiomyoplasty."

A series of experiments carried out by Capouya and coworkers ${ }^{7}$ and by our group ${ }^{13}$ confirmed that adynamic cardiomyoplasty can delay ventricular dilatation and sustain ejection fraction in a canine model of rapid-pacing heart failure. Rapid pacing is a well-defined experimental model that leads to severe dilated cardiomyopathy and heart failure in 3 to 4 weeks. ${ }^{14}$

How can the passive presence of wrapped skeletal muscle prevent cardiac dilatation without constriction? The pericardial sac is known to restrict acute cardiac dilatation such as that induced by a rapid increase in preload. However, it shows virtually no restraining effect on chronic cardiac dilatation. It was proposed that elastic stretchability of the skeletal muscle allowed it to provide dynamic constraint against progressive cardiac dilatation. ${ }^{12}$ We therefore attempted to compare adynamic cardiomyoplasty with cardiac binding using Marlex mesh, which does not share such characteristics of the skeletal muscle wrap.

Vaynblat and associates ${ }^{15}$ studied the cardiac binding effect with polytetrafluoroethylene* in a canine model of doxorubicin-induced heart failure and showed that cardiac binding reduced ventricular dilatation, as we saw in our animals. They did not demonstrate improvement in cardiac function. We also observed better preservation of ventricular volume in the cardiac binding and adynamic cardiomyoplasty animals compared with that in the control group. In addition, we found that ejection fraction was better preserved with adynamic cardiomyoplasty and cardiac binding with Marlex mesh than in the control group. Although not always reaching statistical significance owing to the relatively small sample size, our data indicate a trend that the effects of cardiac binding with Marlex mesh on changes in cardiac dimensions and ejection fraction may be between those observed in the control and in the adynamic cardiomyoplasty groups. Marlex mesh is a synthetic knitted polypropylene monofilament that can stretch somewhat in both directions and induces more fibrotic responses than the polytetrafluoroethylene membrane. Skeletal muscle flap used for adynamic cardiomyoplasty is certainly much more stretchable than these prosthetic membranes. We

${ }^{*}$ Gore-Tex graft, registered trade name of W. L. Gore \& Associates, Inc., Elkton, Md. 
were therefore concerned about the possibility of pericardial constrictions by Marlex mesh and epicardial fibrosis. Thus we carried out left heart catheterization in the cardiac binding group. Our findings of lack of equalization between left and right ventricular end-diastolic pressures in the animals studied suggest that there were no significant restrictions to their ventricular filling. Finally, the mean pulmonary capillary wedge pressures were slightly higher in the cardiac binding group than in the other groups, but this difference did not reach statistical significance. The apparent difference in pulmonary capillary wedge pressure may also reflect some variation in volume status in each animal at the time of study.

Although cardiac binding with Marlex mesh demonstrated its ability to reduce progressive cardiac dilatation and deterioration in ejection fraction during rapid pacing, the effects appeared to be less than those observed with adynamic cardiomyoplasty. We speculate that this is indeed due to the unique characteristics of a living muscle tissue used for cardiomyoplasty. A study on the passive stretch properties of the canine diaphragm muscle suggests a possible advantage for using muscle rather than prosthetic material. ${ }^{16}$ The passive stress-stretch behavior of the muscle was nonlinear, with the muscle getting stiffer as stretch increased. This ability to both yield and support would be a unique advantage in providing passive restraint against left ventricular dilatation without significantly compromising diastolic filling. In contrast, the fibrotic reaction induced by Marlex mesh would provide minimal compliance. In addition to the stretchability, skeletal muscle is known to be able to undergo "conformational change," adapting to chronic changes in resting tension by the addition or deletion of the number of sarcomeres in the muscle fibers. ${ }^{17,}{ }^{18}$ This process allows for the skeletal muscle to restore optimal resting tension, without altering the normal dimension of individual sarcomere units. Although it has not been possible to measure the resting tension of the wrapped muscle per se, serial determination of the Frank-Starling curve in the skeletal muscle ventricle has yielded data consistent with this hypothesis. ${ }^{19}$ By being more versatile in adapting to a changing dimension of the failing heart, cardiomyoplasty can be superior to a prosthetic wrap.

On the other hand, adynamic cardiomyoplasty is a more invasive and complex surgical procedure than cardiac binding with a prosthetic membrane. Dissection of the latissimus dorsi muscle may take 1 to 2 hours, usually done with the patient in the lateral decubitus position. Additional time is required to close and drain the wound and to reposition the patient for sternotomy for the second stage of the procedure, namely, the wrapping of the ventricles with the muscle pedicle that had been inserted into the left pleural cavity. ${ }^{8}$ In part because of such invasiveness and the length of procedure required, dynamic cardiomyoplasty is currently recommended for patients in New York Heart Association Functional class III, rather than in the more seriously ill patients with class IV disease. ${ }^{5}$ In contrast, cardiac binding is a much simpler procedure, which can be carried out rapidly. The procedure lends itself readily to the video-assisted thoracoscopic approach, which makes it even less invasive.

The main benefit of cardiac binding appears to be the process of attenuating the deleterious ventricular remodeling process. Nevertheless, dynamic cardiomyoplasty does offer the added advantage of being able to induce reverse remodeling of dilated cardiomyopathy, as demonstrated clinically. ${ }^{12,20} \mathrm{We}$ believe that passive restraint against further dilatation is not alone sufficient to reverse remodeling and that this may necessitate the added benefit of active systolic assist available through dynamic cardiomyoplasty. The role of cardiac binding in the surgical management of heart failure instead may be as an adjunct to other more definitive procedures, with the simple goal of reducing subsequent progressive dilatation of a failing heart. One such example is to follow Batista's partial ventriculectomy for dilated cardiomyopathy with cardiac binding, thus preventing or delaying the redilatation of the heart. ${ }^{21}$ Although we did not detect deleterious side effects of cardiac binding in this study, both the potential complications and the clinical efficacy of cardiac binding as an adjunct procedure in the management of heart failure require further experimental and clinical explorations.

\section{REFERENCES}

1. Batista RJV, Santos JLV, Takeshita N, Bocchino L, Lima PN, Cunha MA. Partial left ventriculectomy to improve left ventricular function in end-stage heart disease. J Card Surg 1996;11:96-7.

2. Parga JR, Avila LF, Moraes A, Bocchi E, Bacal F, Moreira LF, et al. Partial left ventriculectomy in severe idiopathic dilated cardiomyopathy: assessment of short-term results on shape, and systolic function by magnetic resonance imaging. J Am Coll Cardiol 1997;29(Suppl A):216A.

3. Takeshita N, Kawaguchi AT, Lima PRN, Bocchino L, Verde JL, Batista JVK. Hemodynamic changes in patients under- 
going left ventricular diameter reduction (Batista operation). J Am Coll Cardiol 1997;29(Suppl A):64A.

4. Li CM, Chiu RC-J. Surgical ventricular remodeling: pathophysiological basis for the cardioreduction (Batista) procedure. Heart Failure Rev 1997;2:71-83.

5. Chiu RC-J. Dynamic cardiomyoplasty for heart failure. Br Heart J 1995;73:1.

6. Oh JH, Badhwar V, Chiu RC-J. Mechanisms of dynamic cardiomyoplasty: current concepts. J Card Surg 1996;11: 194-9.

7. Capouya ER, Gerber RS, Drinkwater DC Jr, et al. Girdling effect of nonstimulated cardiomyoplasty on left ventricular function. Ann Thorac Surg 1993;56:867-71.

8. Chiu RC-J. Cardiomyoplasty. In: Edmunds LH Jr, editor. Cardiac surgery in the adult. New York: McGraw-Hill; 1996. p. 1491-504.

9. Furnary AP, Jessup M, Moreira LFP. Multicenter trial of dynamic cardiomyoplasty for chronic heart failure. The American Cardiomyoplasty Group. J Am Coll Cardiol 1996; 28:1175-80.

10. Chen FY, Aklog L, deGuzman BJ, et al. New technique measures decreased transmural myocardial pressure in cardiomyoplasty. Ann Thorac Surg 1995;60:1678-82.

11. Carpentier A, Chachques JC, Acar C, et al. Dynamic cardiomyoplasty at seven years. J Thorac Cardiovasc Surg 1993;106: 42-53.

12. Kass D, Baughman KL, Pak PH, et al. Reverse remodeling from cardiomyoplasty in human heart failure: external constraint versus active assist. Circulation 1995;91:2314-8.
13. Mott BD, Oh JH, Misawa Y, Helou J, Franchischelli D, Chiu RC-J. Mechanisms of cardiomyoplasty: comparative effects of adynamic versus dynamic cardiomyoplasty. Ann Thorac Surg (In press).

14. Wilson JR, Douglas P, Hickey WF, et al. Experimental congestive heart failure produced by rapid ventricular pacing in the dog: cardiac effects. Circulation 1987;75:857-67.

15. Vaynblat M, Chiavarelli M, Shah HR, et al. Cardiac binding in experimental heart failure. Ann Thorac Surg 1997;64:81-5.

16. Strumpf RK, Humphrey JD, Yin FCP. Biaxial mechanical properties of passive and tetanized canine diaphragm. Am J Physiol 1993;265:H469-75.

17. Herring SW, Grimm AF, Grim BR. Regulation of sarcomere number in skeletal muscle: a comparison of hypothesis. Muscle Nerve 1984;7:161-73.

18. Tardieu C, Tabary JC, Tardieu G. Adaptation of sarcomere numbers to the length imposed on the muscle. Adv Physiol Sci 1981;2:99-114.

19. Gealow KK, Bianco RW, Chiu RC-J, et al. Conformational adaptation of muscle: implications in cardiomyoplasty and skeletal muscle ventricles. Ann Thorac Surg 1993;56:520-6.

20. Li CM, Chiu RC-J. The mechanisms and optimization of programming. Volume 1. In: Brachmann J, Stephenson LW, editors. Current clinical practices in dynamic cardiomyoplasty. Armonk [NY]: Futura; 1997. p. 1-51.

21. Moreira LFP, Stolf NFG, Bocchi EA, Bacal F, Bellotti G, Jatene AD. Mid-term results of partial left ventriculectomy in patients with dilated cardiomyopathy. Ann Thorac Surg (In press).

\section{Bound volumes available to subscribers}

Bound volumes of The Journal of Thoracic and Cardiovascular Surgery are available to subscribers (only) for the 1998 issues from the Publisher, at a cost of $\$ 122.00$ for domestic, \$151.94 for Canadian, and \$142.00 for international subscribers for Vol. 115 (January-June) and Vol. 116 (July-December). Shipping charges are included. Each bound volume contains a subject and author index and all advertising is removed. Copies are shipped within 60 days after publication of the last issue of the volume. The binding is durable buckram with the Journal name, volume number, and year stamped in gold on the spine. Payment must accompany all orders. Contact Mosby, Inc., Subscription Services, 11830 Westline Industrial Drive, St. Louis, Missouri 63146-3318, USA; phone $800-453-4351$ or $314-453-4351$.

Subscriptions must be in force to qualify. Bound volumes are not available in place of a regular Journal subscription. 